\title{
Total sacrectomy and Galveston L-rod reconstruction for malignant neoplasms
}

\section{Technical note}

\author{
Ziya L. Gokaslan, M.D., Marvin M. Romsdahl, M.D., Ph.D., Stephen S. Kroll, M.D., Garrett L. \\ Walsh, M.D., Theresa A. Gillis, M.D., David M. Wildrick, Ph.D., and Milam E. Leavens, M.D. \\ Departments of Neurosurgery, General Surgery, Reconstructive and Plastic Surgery, Thoracic and \\ Cardiovascular Surgery, and Neuro-Oncology, Physical Medicine and Rehabilitation Service, The \\ University of Texas M. D. Anderson Cancer Center, Houston, Texas
}

Although radical resection is the best treatment for malignant sacral tumors, total sacrectomy for such tumors has been performed in only a few instances. Total sacral resection requires reconstruction of the pelvic ring plus establishment of a bilateral union between the lumbar spine and iliac bone. This technique is illustrated in two patients harboring large, painful, sacral giant-cell tumors that were unresponsive to prior treatment. These patients were treated with complete en bloc resection of the sacrum and complex iliolumbar reconstruction/stabilization and fusion. Surgery was performed in two stages, the first consisting of a midline celiotomy, dissection of visceral/neural structures, and ligation of internal iliac vessels, followed by an anterior L5-S1 discectomy. The second stage consisted of mobilization of an inferiorly based myocutaneous rectus abdominis pedicle flap for wound closure, followed by an L-5 laminectomy, bilateral L-5 foraminotomy, ligation of the thecal sac, division of sacral nerve roots, and transection of the ilia lateral to the tumor and sacroiliac joints. Placement of the instrumentation required segmental fixation of the lumbar spine from L-3 down by means of pedicle screws and the establishment of a bilateral liaison between the lumbar spine and the ilia by using the Galveston L-rod technique. The pelvic ring was then reestablished by means of a threaded rod connecting left and right ilia. Both autologous (posterior iliac crest) and allograft bone were used for fusion, and a tibial allograft strut was placed between the remaining ilia. The patients were immobilized for 8 weeks postoperatively and underwent progressive rehabilitation. At the 1-year follow-up review, one patient could walk unassisted, and the other ambulated independently using a cane. Both patients controlled bowel function satisfactorily with laxatives and diet and could maintain continence but required self-catheterization for bladder emptying. The authors conclude that in selected patients, total sacrectomy represents an acceptable surgical procedure that can offer not only effective local pain control, but also a potential cure, while preserving satisfactory ambulatory capacity and neurological function.

Key Words * total sacrectomy * giant-cell tumors * surgery * reconstruction

Primary sacral neoplasms involving S-1 and S-2 are amenable to complete en bloc resections for possible 
cure. Tumors selected for this operation ideally have a low propensity for metastasis, yet they are symptomatic and unresponsive to noninterventional therapy. These include sacral giant-cell tumors and low-grade malignant tumors such as chondrosarcomas and chordomas. Whereas subtotal resection of the sacrum caudal to the midportion of the S-1 vertebral body does not destabilize the pelvis, total sacrectomy requires establishment of a bilateral union between the lumbar spine and the ilium, as well as reconstruction of the pelvic ring.[2,5] Preservation of lumbar nerve roots allows ambulation; [4] however, urinary bladder, rectosigmoid colon, and sexual function are markedly altered, although manageable with rehabilitation.

\section{SURGICAL TECHNIQUE}

Surgery was performed in two stages with an interval of 21 (Case 1) and 17 days (Case 2) between stages.

\section{Stage I}

The goal of this stage is to mobilize and dissect visceral and vascular structures from the ventral portion of L-5 and the sacrum. A midline celiotomy is performed via an abdominal incision to expose this region, and the rectosigmoid colon is dissected from the presacral fascia to the coccygeal region. The internal iliac arteries and veins, as well as lateral and median sacral vessels, are divided at their origin to allow ample exposure of the first and second anterior sacral nerve roots (Fig. 1 upper left). The common iliac vessels, distal vena cava, and aorta are mobilized after division of small posterior vessels arising from these structures. Lateral dissection of the sacral ala allows identification of the lumbar trunk (L4-5) of the lumbosacral plexus. The sacroiliac joint is identified lateral to these nerve roots, and bilateral partial ventral sacroiliac osteotomy is performed (Fig. 1 upper right). The lumbosacral disc is exposed and removed along with the anterior aspect of the annulus fibrosis. The ventral sacral periosteum is electrocoagulated, and the S1-3 ventral nerve roots are divided at their foramina, if they are not obscured by the tumor.

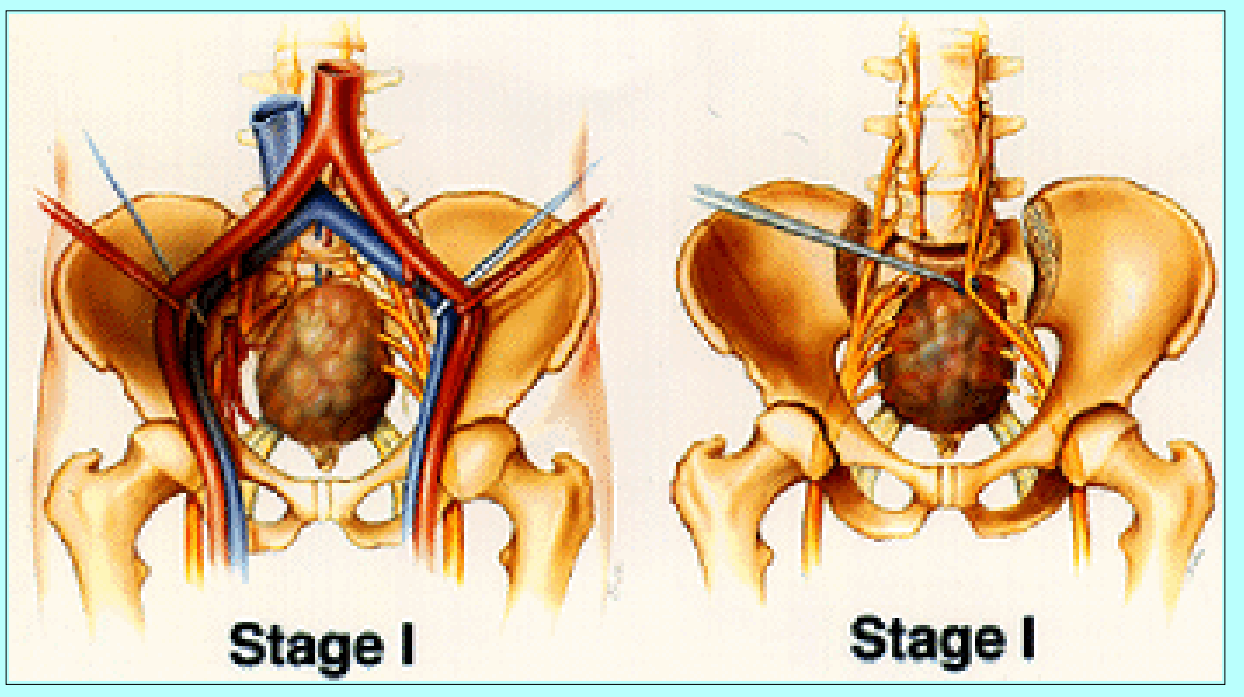

Fig. 1. Artist's renderings depicting the operative fields during Stages I and II of total sacrectomy. Upper Left: Stage I. Ventral view of lumbosacral region after median celiotomy. Inferior vena cava and aorta (and their main branches) are mobilized, allowing a clear view of the L5-S1 disc space and permitting retraction of these vessels for lumbar trunk (L4-5 nerve roots) identification. Median and lateral sacral vessels along with internal hypogastric vessels are ligated. The S-1 foramina and nerve roots are also visualized 
bilaterally; the tumor usually obscures the lower sacral nerve roots (S2-5). Visualized sacral roots are then transected ventrally while preserving the lumbar trunk. Upper Right: Stage I. Ventral view of lumbosacral region with the vessels removed. Nerve roots at S1-3 are transected ventrally. Using a diamond burr, partial (ventral) sacroiliac osteotomy is performed lateral to the lumbar trunk.

A flexible thin silastic sheath is placed dorsal to the vascular structures and the rectum, isolating them from the lumbar vertebrae and sacrum. This inhibits formation of adhesions between these structures and aids in performing the second stage of the operation. After Stage I, a significant ileus lasting approximately 8 days occurs due to interruption of the autonomic presacral nerves.

\section{Stage II}

Stage II is begun by reopening the celiotomy incision. A unilateral myocutaneous pedicle flap fed by the inferior epigastric vessels is mobilized to the pelvic inlet, dorsal to the rectosigmoid colon but ventral to the previously placed silastic sheath (Fig. 1 center left). The abdominal incision is closed, and the patient is placed in the prone-jackknife position for sacral resection via the posterior midline approach.

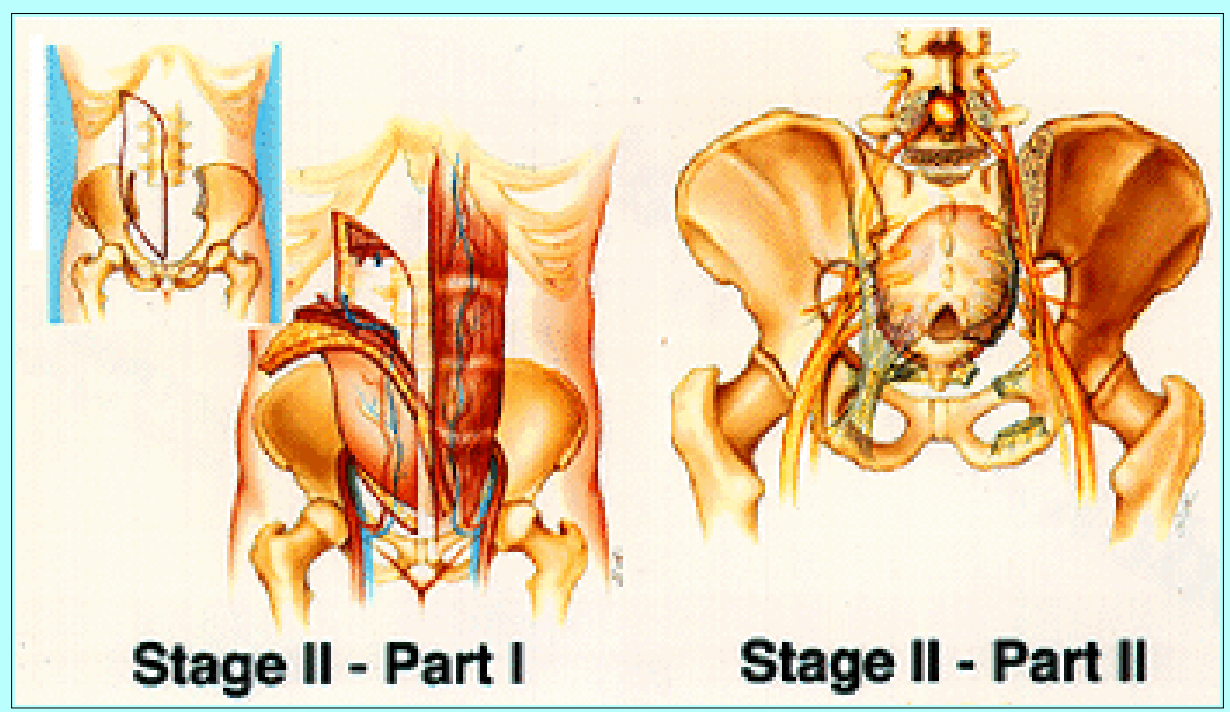

Fig. 1. Center Left: Stage II, Part I. Ventral view of abdominal wall while the patient is supine. A vascularized rectus abdominus muscle with an island of skin and subcutaneous adipose tissue is harvested from the location outlined in inset. Taking care to preserve the inferior epigastric vessels, this flap is placed in the abdominal cavity and the incision is closed. Center Right: Stage II, Part II. Dorsal view of the lumbosacral region while the patient is in the jackknife position after completion of L-5 laminectomy and bilateral L5-S1 foraminotomy. The completed L5-S1 discectomy and dorsal sacroiliac osteotomy are also shown. The thecal sac is ligated distal to the takeoff of the L-5 nerve roots bilaterally.

The posterior incision extends from L-2 to beyond the coccyx, leaving skin, subcutaneous tissue, and muscle in place over the sacrum to facilitate en bloc resection. The posterior iliac crests, greater sciatic foramina, and sciatic nerves are exposed bilaterally, as well as the L3-5 spinous processes, facet joints, and transverse processes. An L-5 laminectomy exposes the thecal sac and cauda equina below this level. Sacral nerve roots are then divided, and the thecal sac is closed with a double layer of sutures. The remaining posterior L5-S1 intervertebral disc is excised, and the posterosuperior iliac spines are removed, facilitating bilateral osteotome cuts lateral to the ala of the sacrum and parallel to the sacroiliac 
joints, thus completing the osteotomy cuts made in these planes during the Stage I procedure (Fig. 1 center right).

Partial mobilization of the sacrum facilitates identification of the sacrospinous and sacrotuberous ligaments, which are then transected. The rectum is dissected from the distal sacrococcygeal attachments. The previously placed silastic sheath, visualized at this point, serves as a guide in this dissection. Sacral nerve roots are divided as they exit the sacrum, protecting the sciatic nerves from injury. The entire sacrum along with the neoplasm is then removed en bloc (Fig. 1 lower left).

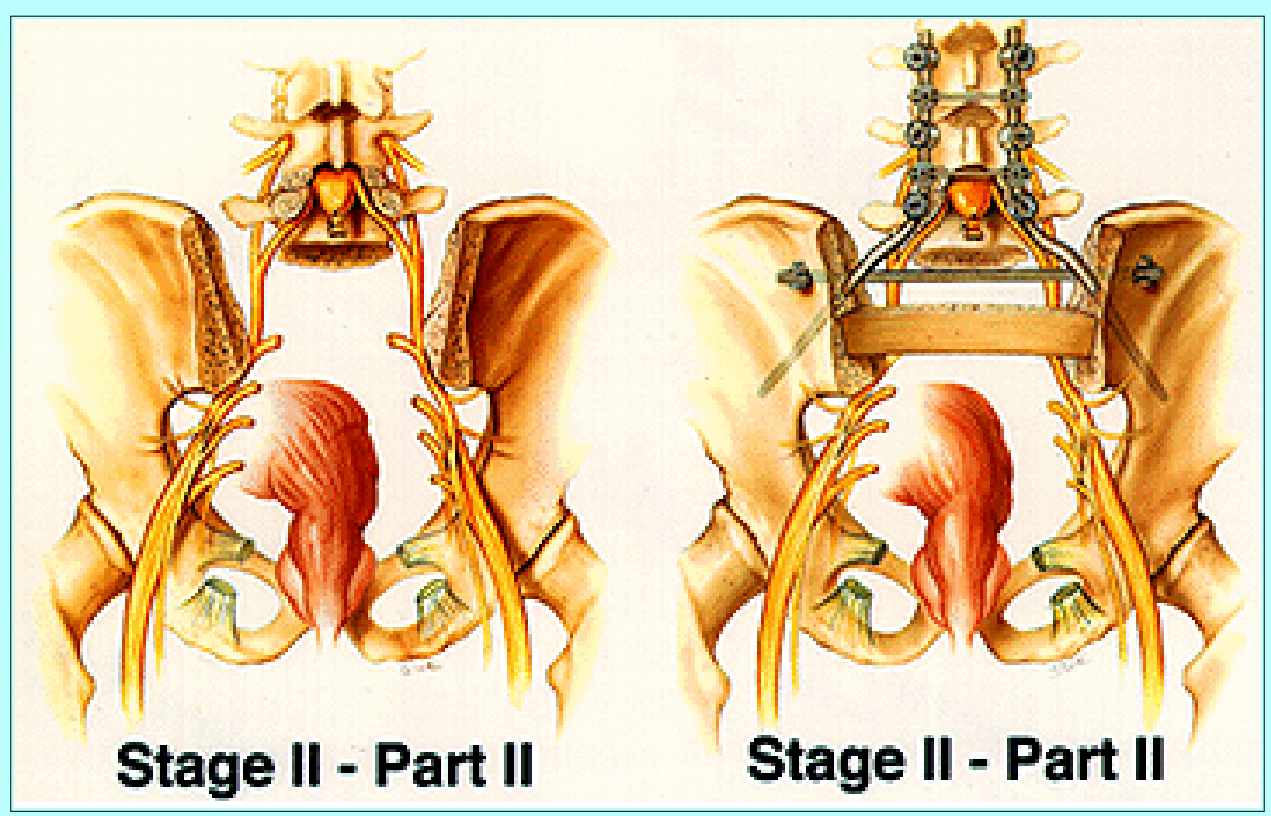

Fig. 1. Lower Left: Stage II, Part II. Dorsal view of lumboiliac region after en bloc removal of sacrum. The cut ends of nerve roots S1-4 are shown bilaterally, as well as the bilaterally preserved lumbar trunk and remaining sciatic nerve. Sacrotuberous and sacrospinous ligament cut ends are also depicted. The dorsal rectal wall is viewed ventrally. Lower Right: Stage II, Part II. Dorsal view of the lumbosacral region after reconstruction. Pedicle screws are seen bilaterally at L3-5 along with rods that are attached to the screws and bent (L-shaped) and embedded between the cortices of the remaining ilia as reported in the Galveston technique. A threaded rod (transiliac bar) is placed ventral to the Galveston rods but dorsal to the lumbar trunk, and the ends outside the iliac cortical surface are secured with $\mathrm{C}$-shaped clamps. The tibial allograft is seen bridging the defect between the remaining ilia.

\section{Reconstruction of the Sacrum}

Two vertical L-shaped rods are positioned bilaterally in a manner allowing fixation to the L3-5 pedicles on each side, according to the Galveston technique.[1] Two to three cross-connecting rods are used to secure the vertical rods to each other. Distally, the vertical rods are directed laterally into the ilium between the two cortices. A transverse threaded rod (transiliac bar) is placed to fix opposing iliac bones to each other and thereby prevent axial rotation of the lumboiliac union (Fig. 1 lower right). Both autologous (from posterior iliac crests) and allogeneic bone grafts are placed to promote fusion of the transverse processes and lamina from L-3 distally to the medioposterior aspect of the transected ilium bilaterally. A tibial allograft strut is used to close the space between the two ilia, and a bone fusion promoter (Grafton) and bone chips (allograft) are added across the graft area to facilitate fusion of the 


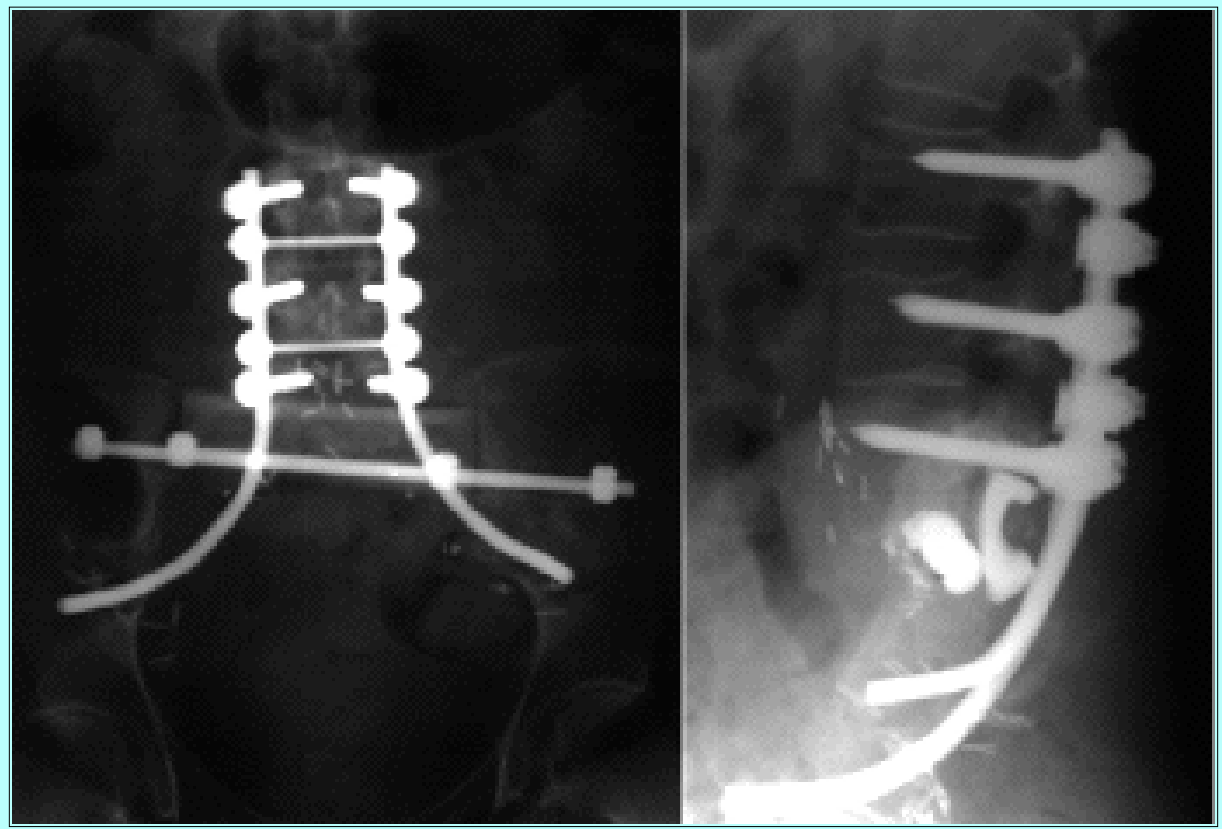

Fig. 2. Postoperative anteroposterior/lateral lumbosacral $\mathrm{x}$-ray film showing iliolumbar reconstruction performed in the patient in Case 2. At levels L3-5, pedicle screws are visible connected by L-rods embedded bilaterally in iliac wings as described in the Galveston technique. The transiliac threaded rod and tibial allograft can also be seen crossing the defect between the ilia.

The previously mobilized rectus abdominis myocutaneous pedicle flap is advanced through the pelvis, positioned over the reconstructed area, and secured to gluteus muscles and skin after placement of closed drains.

\section{Rehabilitation Procedures and Clinical Outcomes}

Mobilization. Patients remain at bed rest for 8 weeks before the initiation of bed mobility therapy, transfer functions, and sitting, eventually progressing to parallel bars and ambulation with a rolling walker. Postoperative weakness is present in hip extensors and plantar flexors of the feet. However, with physiotherapy and time, the patients' gait improves markedly.

Bowel and Bladder Programs. A neurogenic bowel elimination program is initiated, consisting of a moderately constipating diet together with regularly timed laxatives and evacuations. The neurogenic lower motor neuron bladder with flaccid external sphincter is managed by intermittent catheterization at 6-hour intervals. Voluntary bowel and bladder control is permanently lost due to sectioning of both S-2 nerve roots, which principally control sphincter function.[4] Bowel elimination has been optimized by a strict program, and training of patients in self-catheterization of the bladder has been achievable and accepted.

Pain Management. The severe preoperative pain is relieved. Postoperative sacral neurogenic pain is initially controlled with intravenously administered narcotic drugs, followed by gradual replacement with oral analgesic and nonsteroidal antiinflammatory medications.

\section{ILLUSTRATIVE CASES}


Two patients, each with localized primary giant-cell tumors of the sacrum, were considered candidates for total sacral resection because of the following conditions: 1) intractable pain; 2) impairment of sacral nerve functions; 3 ) localized progressive disease; and 4) the absence of effective noninterventional treatments.

\section{Case 1}

This 37-year-old woman had previously developed severe pain in the buttocks and lower back associated with altered bladder function. A highly vascular sacral giant-cell tumor was identified, involving the entire sacrum distal to S-2. In June 1994, a sacral laminectomy with partial removal of tumor, decompression of nerve roots, and radiation therapy afforded relief for 6 months, after which her symptoms returned in the form of severe pain, perineal numbness, constipation, and episodes of bowel and bladder incontinence.

At this time, no subjectively observed gait change or motor weakness was evident. However, sensory loss was present in the perineal and perianal regions and in the posterior right thigh. A magnetic resonance (MR) image revealed a large infiltrating sacral midline mass extending into the S- 1 vertebral body, both sacral alae, sacral lamina with posterior sacral ligaments, sacral canal at the level of the S-1 vertebra, and a major portion of the right sacroiliac joint. A successful total sacrectomy was performed: Stage I on May 16, 1995, and Stage II on June 6, 1995. Blood loss for Stage I was estimated at 5.5 L, and the operating time was 19.75 hours. Stage II of the operation required the transfusion of $16 \mathrm{~L}$ of blood and was completed in 14.5 hours. Although great effort was made intraoperatively to spare the L-5 nerve roots in this patient, her postoperative neurological evaluation (including an electromyography) showed L-5 denervation. Despite this, at 1-year follow-up review, this patient was able to walk with the use of a cane.

\section{Case 2}

This 30-year-old woman had previously developed pain in the lower back and left lower extremity, and MR imaging showed a tumor filling the sacral canal. In February 1995 she underwent lumbar laminectomy for nerve root decompression that provided pain relief for 6 weeks, after which bilateral leg and intense pelvic pain recurred. Her symptoms were unresponsive to physical therapy and steroid administration.
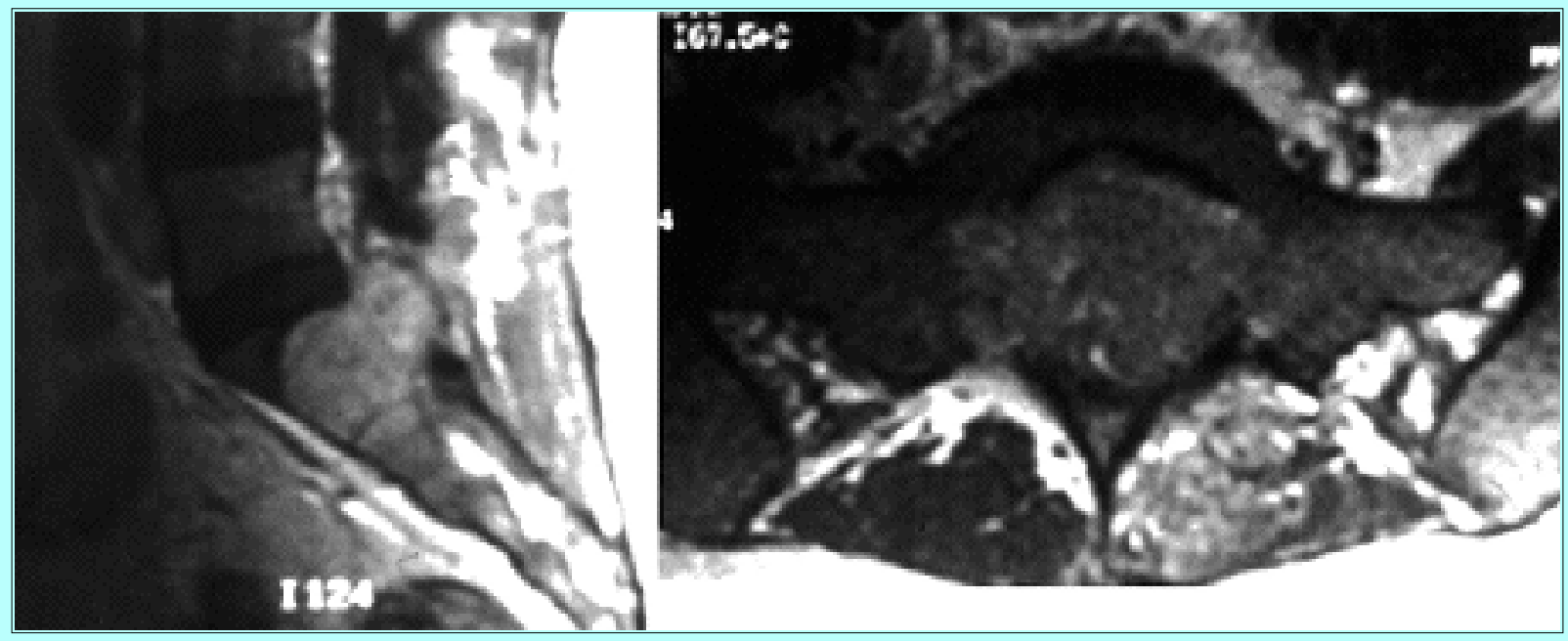

Fig. 3. Preoperative MR image obtained in one patient, revealing a recurrent giant-cell tumor involving the entire sacrum, extending rostrally to the L5-S1 disc space and dorsally 
filling the spinal canal. The patient presented with intractable local pain, bilateral sciatica, and worsening bowel/bladder dysfunction.

The patient developed sensory loss in the perineum, buttocks, and posterior thigh, along with decreased rectal sphincter tone, although muscle functions were not altered. An MR image revealed an extensive giant-cell tumor ranging from the lumbosacral junction to the coccyx, including the L5-S1 disc space dorsally, complete obliteration of the sacral canal, and extension of the tumor into dorsal soft tissues (Fig. 3). A successful total sacrectomy was performed: Stage I on June 27, 1995, and Stage II on July 14, 1995. The operating time for Stage I was 11.5 hours, and $2.7 \mathrm{~L}$ of blood was required. In Stage II the blood loss was estimated at $19 \mathrm{~L}$, and the operating time was 22.5 hours. At the 1-year follow-up review, this patient was walking unassisted (Fig. 4) and was able to drive a car.

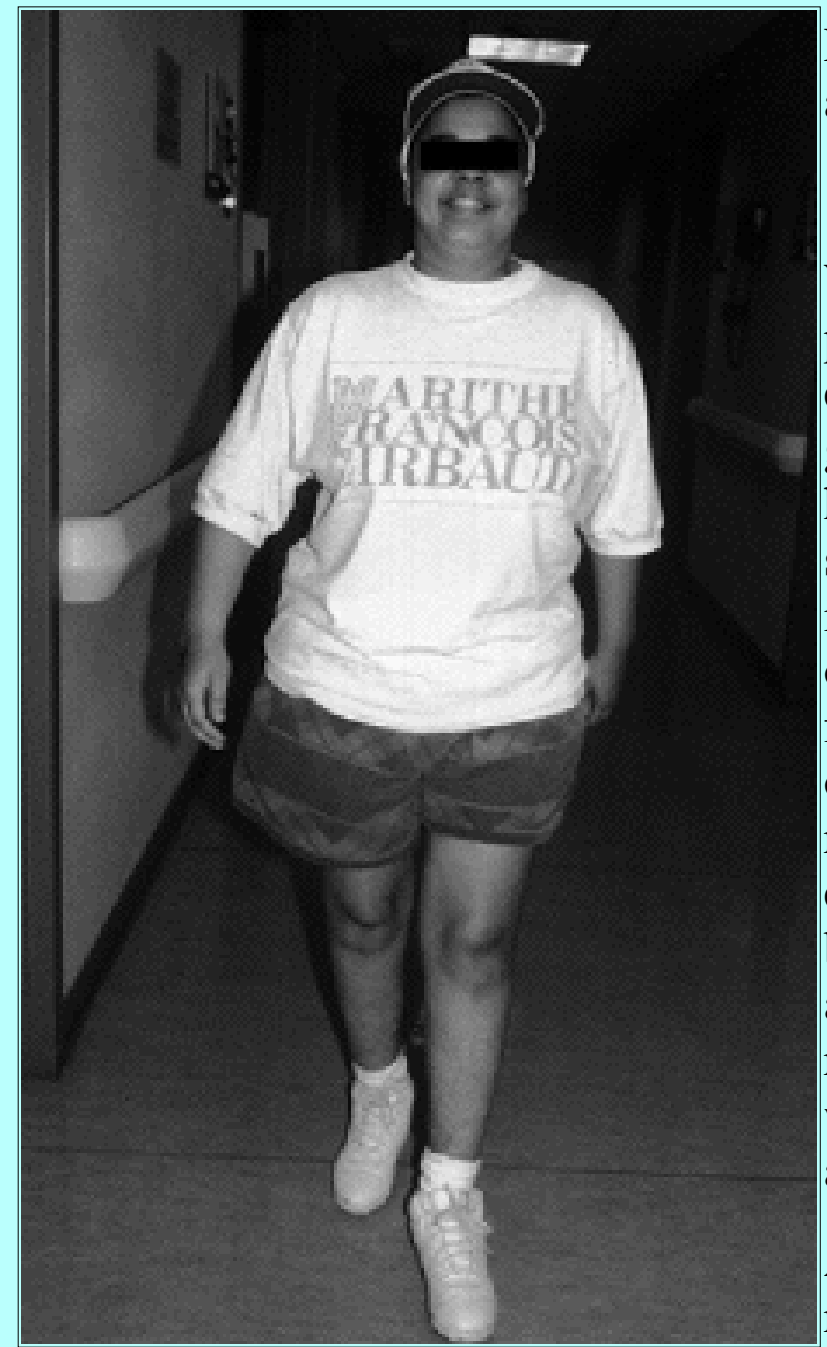

Fig. 4. Photograph showing that the patient in Case 2 is able to walk unassisted.

\section{DISCUSSION}

We consider radical resection to be the best treatment for primary malignant tumors involving the entire sacrum. This is especially true for aggressive sacral neoplasms such as giant-cell tumors that have a low tendency to metastasize but a high resistance to nonsurgical treatment. However, total sacrectomy for such tumors has been performed in only a few instances. $[2,3,6-8,10]$ This is in part because of the complexity of the operation, which requires the participation of specialists from several areas including neurosurgery, general surgery, orthopedic surgery, and plastic surgery. The total sacrectomies reported by Edwards[3] and Michel[6] did not include any efforts at reconstruction of the pelvic ring or of connections between the spine and pelvis. We believe that reconstruction after total sacrectomy is necessary to achieve the stabilization required for ambulation and that it is likely to permit patients to walk sooner during rehabilitation and resume their daily activities earlier.

A variety of instrumentation has been used previously for reconstruction after total sacrectomy. From a biomechanical standpoint these devices progress in their level of sophistication as follows: the use of sacral bars spanning the ilia and connected to the spine with Harrington rods and hooks; [8] the use of the more advanced Cotrel-Dubousset rods and hooks with the sacral bars (or AO plates); $[7,10]$ the use of transpedicular screws (and an internal spinal fixator) with iliac screws connected by plates;[2] and the use of vertical Galveston rods attached to cross-connecting spinal rods, plus a threaded transiliac rod (as described in this paper).

The total sacrectomy reconstruction procedure used in two patients by Shikata, et al.,[8] included two sacral bars spanning both ilia (with the more anterior one passing through the body of L-5), Harrington rods and hooks for spinal instrumentation, and the use of massive bone grafts to promote fusion between 
the lumbar spine and pelvis. A problem with this construct is that the sacral bars connect the posterior iliac wings, which are soft and do not provide firm fixation;[9] moreover, this reconstruction fails to confer much axial rotational stability on the spinal column. Tomita and Tsuchiya[10] performed total sacrectomies in three patients, including reconstructions paralleling those of Shikata, et al.,[8] by using two or three sacral rods (or two AO plates) for bilateral fixation of the iliac wings, either Harrington or Cotrel-Dubousset spinal instrumentation (vertical rods and hooks), and large amounts of autograft and allograft (fibula) bone. Although Cotrel-Dubousset is superior to Harrington spinal instrumentation, this reconstructive effort suffers from the same limitations as that of Shikata, et al.,[8] for iliac wing fixation and axial rotational stability.

Santi, et al.,[7] substituted two large Steinmann pins for the sacral bars used in the two previous reports $[8,10]$ to connect the ilia in a patient after total sacrectomy. They also used Cotrel-Dubousset rods and hooks to attach the spine to the horizontal (sacral) pins and used only autograft iliac bone between L-5 and both ilia. The more proximal transverse pin had to be removed at 5 months postsurgery because it became dislodged when the patient fell, and at 7 months postsurgery all hardware was removed, and additional autograft and allograft bone were placed between L-4 and each ilium because of delayed L5-iliac fusion. This result may illustrate the importance of using sufficient spinal-iliac bone grafting to augment the instrumentation.

Curiously, in the most recent report of a reconstruction following total sacrectomy,[2] no mention was made of bone grafting to supplement the instrumentation, which consisted of an internal spinal fixing device attached to vertebrae L-3 and L-4 by transpedicular Schanz screws, with the device attached to two 65-mm iliac screws (one implanted in each ilium) connected to each other by iliac plates. An additional pair of Schanz screws was implanted in each ilium proximal to the iliac plates and cross-connected via "normal blocks." This arrangement avoids the use of Harrington or Cotrel-Dubousset hook and rod systems connecting to sacral bars, because such hooks can accidentally disengage from vertebrae and sacral bars, and it may provide more axial stability than was seen in earlier efforts. However, the lack of transverse sacral bars may not prevent the ilia, which are firmly anchored posteriorly, from spreading in a lateral anterior direction in the so-called "open-book" phenomenon.

We consider the Galveston L-rod technique[1] used in this study to be the optimum reconstructive method after total sacrectomy and to represent a sort of evolutionary endpoint to the others discussed earlier. Each Galveston rod forms a one-piece bridge between the lumbar spine and an ilium, and the transiliac rod completes the pelvic ring. The instrumentation is more sophisticated in that the two L-shaped rods are segmentally fixed bilaterally to the lumbar spine (L3-5 pedicles) with pedicle screws and to each other with two to three cross-connecting rods, and each L-rod is then embedded laterally between the iliac cortices. Augmented by both autologous and allogeneic bone grafts, this instrumentation system prevents both downward (caudal) migration and axial rotation of the spinal column, while simultaneously preventing the open-book phenomenon with the use of a threaded transiliac rod placed more anteriorly on the ilium, which avoids slippage associated with anchorage in the softer posterior ilium. This system provides stability around the horizontal axis of the spinal column while also preventing rotation around this axis.

It is interesting to compare the results of this study with those of other reports of total sacrectomy regarding length of the operation, blood loss sustained, and outcome (Table 1). Blood loss from total sacrectomy can be enormous, as seen in the range of values (6.5-40 L for single-stage operations; 10.1-80 L for two-stage operations) presented in Table 1. Our estimation of the blood loss for our two 
total sacrectomy cases was somewhat higher than that reported for single-stage operations, except for Blatter, et al.,[2] (40 L) and the multistage operations of Shikata, et al.,[8] but our total operating time (34 hours) is also substantially longer than that reported by others, even for three-stage operations. [8] Perhaps more significantly, of 10 patients undergoing total sacrectomy (Table 1), only two were able to walk unassisted by a cane: one at 5.75 years postsurgery[10] and one at 1 year postoperatively (our Case 2). Of three patients able to walk with a cane and no braces postsurgery, two did so after 2 to 2.75 years, $[8,10]$ and one was able to do so at 1 year postoperatively (our Case 1 ). Because the other five patients walking with and without canes (but no braces) were all in their 30s, this indicates a potentially shorter recuperation and a more favorable outcome resulting from the surgical method we present here.

\begin{tabular}{|c|c|c|c|c|c|}
\hline \multicolumn{6}{|c|}{$\begin{array}{c}\text { TABLE } 1 \\
\text { REVIEW OF LITERATURE COMPAR NG OPERATING TIME, ESTMMTED BLOOD LOSS, AND PATIENT } \\
\text { OUTCOME FOR TOTAL SACRECTOMN }\end{array}$} \\
\hline Authors \& Year & $\begin{array}{l}\text { Age }(y s) \\
\text { Sex }\end{array}$ & $\begin{array}{l}\text { No } \\
\text { of Op } \\
\text { Stages }\end{array}$ & $\begin{array}{l}\text { Total } \\
\text { op Time } \\
\text { (hrs) }\end{array}$ & $\begin{array}{l}\text { Total } \\
\text { Blood } \\
\text { Loss (L) }\end{array}$ & Outcome \\
\hline Michel, 1990 & $27, \boldsymbol{M}$ & 2 & 20 & 80 & $\begin{array}{l}\text { walks } w / 2 \text { ankle orthoses } \& \\
2 \text { canes ( }=2 \text { yrs) }\end{array}$ \\
\hline Shikata, et al., 1988 & $34, M$ & 3 & 26 & 8.3 & $\begin{array}{l}\text { walks w/short trace plus cane; } \\
\text { crefers wheelchaj } 2.16 \text { yrs) }\end{array}$ \\
\hline $\begin{array}{l}\text { Tomita \& Tsuchiya, } \\
1990\end{array}$ & $\begin{array}{l}36, F \\
32, F \\
36, M\end{array}$ & $\begin{array}{l}2 \\
1 \\
1\end{array}$ & $\begin{array}{l}23 \\
10 \\
19.5\end{array}$ & $\begin{array}{r}10.1 \\
7.5 \\
8.4\end{array}$ & $\begin{array}{l}\text { walks w/cane ( } 22 \text { yrs) } \\
\text { walks wo cane }(5.75 \text { yrs) } \\
\text { malks w/cane; prefers wheel- } \\
\text { chair ( } 2.75 \text { yrs) }\end{array}$ \\
\hline & $72, M$ & 1 & 13 & 17 & $\begin{array}{l}\text { both ankles unstable; walks in } \\
\text { ankle-foot orthoses ( } 10 \text { mos) }\end{array}$ \\
\hline Santi, etal., 1993 & $48, M$ & 1 & 18 & 6.5 & $\begin{array}{l}\text { instrumentation remo'ed } \& \\
\text { bone graft added between L-4 } \\
\& \text { both ilia }(7 \text { mos); walks } w / \\
\text { tilat dynam ic orthoses } \\
\text { (2.75 yrs) }\end{array}$ \\
\hline Eatter, et al., 1994 & $43, M$ & 1 & not stated & 40 & $\begin{array}{l}\text { walks wicrutches for } 30 \mathrm{~min} \\
\text { (2 yrs) }\end{array}$ \\
\hline $\begin{array}{l}\text { Gokasian, et al. } \\
\text { (present study) }\end{array}$ & $\begin{array}{l}37, \mathrm{~F} \\
30, \mathrm{~F}\end{array}$ & $\frac{2}{2}$ & $\begin{array}{l}34.2 \\
34\end{array}$ & $\begin{array}{l}21.5 \\
21.7\end{array}$ & $\begin{array}{l}\text { walks w/cane (1 yr) } \\
\text { walks unassisted \& dri ves a car } \\
\text { (1 yr) }\end{array}$ \\
\hline
\end{tabular}

\section{CONCLUSIONS}

We believe that total sacral resection is a feasible operation for primary malignant tumors involving the entire sacrum. In selecting patients for this procedure one considers histological findings, management options, patient motivation, performance status, and freedom from metastatic disease. Reconstruction of the union between the lumbar spine and the ilia with metallic devices achieves stabilization suitable for ambulation. Preservation of lumbar (L-5) nerve roots allows ambulation; [4] however, urinary bladder, rectosigmoid colon, and sexual function are markedly altered, although they are manageable with rehabilitation. Optimum management of patients undergoing total sacral resection includes participation of treatment team members from different specialties.

\section{Acknowledgment}

We thank Dr. Weiming Shi for help in preparing the figures. 


\section{References}

1. Allen BL Jr, Ferguson RL: The Galveston technique for L rod instrumentation of the scoliotic spine. Spine 7:276-284, 1982

2. Blatter G, Ward EGH, Ruflin G, et al: The problem of stabilization after sacrectomy. Arch Orthop Trauma Surg 114:40-42, 1994

3. Edwards CC: Spinal reconstruction in tumor management, in Uhthoff HK (ed): Current Concepts of Diagnosis and Treatment of Bone and Soft Tissue Tumors. Berlin: Springer-Verlag, 1984, pp 329-349

4. Fujimura Y, Maruiwa H, Takahata T, et al: Neurological evaluation after radical resection of sacral neoplasms. Paraplegia 32:396-406, 1994

5. Gunterberg B, Romanus B, Stener B: Pelvic strength after major amputation of the sacrum. An experimental study. Acta Orthop Scand 47:635-642, 1976

6. Michel A: Total sacrectomy and lower spine resection for giant cell tumor: one case report. Chir Organi Mov 75 (Suppl 1): 117-118, 1990

7. Santi MD, Mitsunaga MM, Lockett JL: Total sacrectomy for a giant sacral schwannoma. A case report. Clin Orthop 284:285-289, 1993

8. Shikata J, Yamamuro T, Kotoura Y, et al: Total sacrectomy and reconstruction for primary tumors. J Bone Joint Surg (Am) 70:122-125, 1988

9. Thomson J, Doty JR: Sacral biomechanics and reconstruction, in Doty JR, Rengachary SS (eds): Surgical Disorders of the Sacrum. New York: Thieme Medical Publishers, Inc, 1994, pp 253-256 10. Tomita K, Tsuchiya H: Total sacrectomy and reconstruction for huge sacral tumors. Spine 15:1223-1227, 1990

Manuscript received April 1, 1997.

Accepted in final form June 4, 1997.

Address reprint requests to: Ziya L. Gokaslan, M.D., Department of Neurosurgery, Box 64, The University of Texas M. D. Anderson Cancer Center, 1515 Holcombe Boulevard, Houston, Texas 77030. 